\title{
Expression of osteoprotegerin from a replicating adenovirus inhibits the progression of prostate cancer bone metastases in a murine model
}

\author{
James J Cody ${ }^{1, *}$, Angel A Rivera ${ }^{2, *, \dagger}$, Gray R Lyons ${ }^{1, \ddagger}$, Sherry W Yang ${ }^{1}$, Minghui Wang ${ }^{2}$, Jason W Ashley ${ }^{1}$, \\ Sreelatha Meleth ${ }^{3, \S}$, Xu Feng ${ }^{1}$, Gene P Siegal ${ }^{1,4,5}$ and Joanne T Douglas ${ }^{2,5}$
}

\begin{abstract}
Metastatic involvement of the skeleton is a frequent consequence of advanced prostate cancer. These skeletal metastases cause a number of debilitating complications and are refractory to current treatments. New therapeutic options are being explored, including conditionally replicating adenoviruses (CRAds). CRAds are engineered to selectively replicate in and destroy tumor cells and can be 'armed' with exogenous transgenes for enhanced potency. We hypothesized that a CRAd armed with osteoprotegerin (OPG), an inhibitor of osteoclastogenesis, would inhibit the progression of prostate cancer bone metastases by directly lysing tumor cells and by reducing osteoclast activity. Although prostate cancer bone metastases are predominantly osteoblastic in nature, increased osteoclast activity is critical for the growth of these lesions. Ad5- $\Delta 24-\mathrm{sOPG}-\mathrm{Fc}-\mathrm{RGD}$ is a CRAd that carries a fusion of the ligand-binding domains of OPG and the Fc region of human IgG1 in place of the viral E3B genes. To circumvent low tumor cell expression of the native adenoviral receptor, an arginine-glycine-aspartic acid (RGD) peptide insertion within the viral fiber knob allows infection of cells expressing $\alpha_{v}$ integrins. A 24-base pair deletion $(\Delta 24)$ within viral E1A limits replication to cells with aberrant retinoblastoma cell cycle regulator/tumor suppressor expression. We have confirmed that Ad5- $\Delta 24-\mathrm{sOPG-Fc-RGD} \mathrm{replicates} \mathrm{within} \mathrm{and} \mathrm{destroys}$ prostate cancer cells and, in both murine and human coculture models, that infection of prostate cancer cells inhibits osteoclastogenesis in vitro. In a murine model, progression of advanced prostate cancer bone metastases was inhibited by treatment with Ad5- $\Delta 24-\mathrm{SOPG}-\mathrm{Fc}-\mathrm{RGD}$ but not by an unarmed control CRAd.
\end{abstract}

Laboratory Investigation (2013) 93, 268-278; doi:10.1038/labinvest.2012.179; published online 28 January 2013

KEYWORDS: adenovirus; bone metastasis; oncolytic virus; osteoprotegerin; prostate cancer; virotherapy

Advanced prostate cancer exhibits a propensity for metastasis to the skeleton, and thus a majority of patients with late-stage disease will be diagnosed with bone metastases. ${ }^{1,2}$ The growth of metastatic cells in bone disrupts normal bone physiology and structure ${ }^{3}$ and causes a range of serious complications, including pain, pathological fractures and spinal cord compression. $^{2,4}$ Current treatments with surgery, radiotherapy, chemotherapy and bisphosphonate administration may slow disease progression, but are associated with deleterious side effects ${ }^{5}$ and are often not curative. In light of the above, new therapies for this disease are urgently needed.

One new class of anticancer agents is comprised of conditionally replicating adenoviruses (CRAds) based upon human serotype $5 .^{6}$ These are adenoviruses that have been engineered to selectively replicate within cancer cells, thereby amplifying the input dose of virus and destroying the infected tumor cells by lysis. Through multiple rounds of selective infection, replication, lysis and spread, CRAds have the potential to destroy tumors while sparing normal tissue.

\footnotetext{
'Department of Pathology, The University of Alabama at Birmingham, Birmingham, AL, USA; ${ }^{2}$ Division of Human Gene Therapy, Departments of Medicine, Obstetrics and Gynecology, Pathology and Surgery, The University of Alabama at Birmingham, Birmingham, AL, USA; ${ }^{3}$ Division of Preventive Medicine, Department of Medicine, The University of Alabama at Birmingham, Birmingham, AL, USA; ${ }^{4}$ The Center for Metabolic Bone Disease Core Laboratory, The University of Alabama at Birmingham, Birmingham, AL, USA and ${ }^{5}$ The Gene Therapy Center, The University of Alabama at Birmingham, Birmingham, AL, USA

Correspondence: Dr GP Siegal, MD, PhD, Department of Pathology, Division of Anatomic Pathology, The University of Alabama at Birmingham, 50820 th Street South, HSB 149K, Birmingham, AL 35294, USA.

E-mail: gsiegal@uab.edu

*These authors contributed equally to this work.

†Present address: Department of Pediatrics, Division of Hematology and Oncology and The Gene Therapy Center, Emory University, Atlanta, GA, USA.

¥Present address: Medical Scientist Training Program, Duke University, Durham, NC, USA.

SPresent address: Statistics and Epidemiology Division, Social Statistical and Environmental Sciences, RTI International, Atlanta, GA, USA.

Received 12 July 2012; revised 8 November 2012; accepted 29 November 2012
} 
However, clinical trials have shown that while CRAds are safe to administer, ${ }^{7}$ their potency must be improved before the full potential of this treatment modality can be realized.

One strategy to increase the efficacy of a CRAd is to employ it as a platform for the delivery of a therapeutic transgene. Owing to viral replication, an 'armed' CRAd amplifies the input dose of the transgene and can exert an antitumor effect by multiple mechanisms of action. A variety of armed CRAds directed against a range of malignancies has been described, and it is clear that the inclusion of a rationally selected transgene enhances the potency of a CRAd. ${ }^{8}$ An armed CRAd intended for prostate cancer bone metastasis will therefore be most effective when it has been selected in consideration of tumor-bone interactions. Prostate cancer bone metastases involve a disruption of normal bone homeostasis and influence the bone microenvironment in ways that are not fully understood. Prostate cancer cells produce a variety of factors including bone morphogenetic proteins, endothelin 1, and insulin-like growth factors that induce the growth of lesions, which are predominantly osteoblastic in their behavior. ${ }^{9}$ However, both blastic and lytic processes are involved, and thus osteoclasts also contribute to lesion growth. ${ }^{10}$ Prostate cancer cells can mediate osteoclast formation both directly and indirectly, ${ }^{11}$ largely through the interaction of receptor activator of NF- $\mathrm{KB}$ ligand (RANKL) on osteoblasts with its receptor RANK on osteoclast precursors. It has been shown that blockade of the RANK/RANKL interaction inhibits the progression of prostate cancer bone metastases, ${ }^{12}$ even those which are osteoblastic in nature. ${ }^{13,14}$ This interaction can be disrupted by the normal bone protein osteoprotegerin (OPG), which is a soluble decoy receptor for RANKL. ${ }^{15,16}$ OPG is secreted by osteoblasts and bone stromal cells as a key mediator of normal bone homeostasis; it prevents the binding of RANKL with RANK to inhibit osteoclast differentiation/activation and promote bone formation. Several studies have demonstrated that OPG inhibits the progression of prostate cancer bone metastases. ${ }^{13,17-21}$ We therefore hypothesized that a CRAd armed with OPG would reduce the growth of prostate cancer bone metastases by two means: direct lysis of tumor cells due to viral replication, and a reduction in tumor burden by the inhibition of osteoclastic bone resorption by OPG.

We have previously constructed and described the armed CRAd used in this study, designated Ad5- $\Delta 24-s O P G-F c-$ RGD. ${ }^{22}$ Cancer-selective replication is conferred by means of a 24-base pair deletion in the $E 1 \mathrm{~A}$ gene, ${ }^{23}$ which yields a protein unable to bind and inactivate the retinoblastoma tumor suppressor/cell cycle regulatory protein and restricts efficient viral replication to neoplastic cells. To enhance tumor cell transduction, this armed CRAd also includes a fiber knob with an arginine-glycine-aspartic acid (RGD) peptide insertion in the HI loop. ${ }^{24}$ This modification directs initial binding of the virus to $\alpha_{\mathrm{v}} \beta_{3}$ and $\alpha_{\mathrm{v}} \beta_{5}$ integrins, which are involved in prostate cancer bone metastasis, ${ }^{25,26}$ and thus overcomes the deficiency of the native coxsackievirus and adenovirus receptor (CAR) on prostate cancer cells. ${ }^{27}$ This armed CRAd carries a transgene encoding the RANKLbinding domains of OPG fused to the Fc portion of human IgG1. It therefore lacks the domains of OPG that bind tumor necrosis factor-related apoptosis-inducing ligand, ${ }^{28}$ precluding its ability to act as a survival factor for prostate cancer cells. ${ }^{29}$ We have previously shown that the expression of OPG-Fc does not alter the selectivity of replication of the parent CRAd in experiments involving normal human epithelial cells and human liver slices. ${ }^{22}$

\section{MATERIALS AND METHODS Cells}

The human prostate cancer cell lines $\mathrm{LNCaP}^{30,31}$ and $\mathrm{PC}^{32}$ were purchased from the American Type Culture Collection (ATCC; Manassas, VA, USA). The human prostate cancer cell line $\mathrm{C} 4-2 \mathrm{~B}$, a subline of $\mathrm{LNCaP}$ with enhanced propensity for bone metastasis, was a gift from Dr Leland Chung. ST2 murine bone marrow stromal cells ${ }^{33}$ were from the Riken Cell Bank, Japan. ST2 cells were propagated in $\alpha$-minimum essential medium ( $\alpha$-MEM) and both the LNCaP and PC3 prostate cancer cells were cultured in Roswell Park Memorial Institute (RPMI) 1640 medium. C4-2B cells were cultured in T-Medium (Invitrogen, Carlsbad, CA, USA). These media were supplemented with $10 \%(\mathrm{v} / \mathrm{v})$ heat-inactivated fetal bovine serum (FBS; Invitrogen), L-glutamine $(2 \mathrm{mM})$, penicillin $(100 \mathrm{U} / \mathrm{ml})$ and streptomycin $(100 \mu \mathrm{g} / \mathrm{ml})$. All cell lines were cultured at $37^{\circ} \mathrm{C}$ in a humidified atmosphere, with ST2 cells maintained at $8 \% \mathrm{CO}_{2}$ and all others at $5 \% \mathrm{CO}_{2}$. Except where otherwise noted, media and supplements were from Mediatech (Herndon, VA, USA).

A C4-2B cell subline that stably expresses luciferase (C4-2B-LUC) was generated by transduction of the cells with a lentiviral vector encoding the firefly luciferase gene, as follows. 293GPG cells were cultured in Dulbecco's Modified Eagle Medium supplemented with 10\% (v/v) heat-inactivated FBS, tetracycline, puromycin, G418, and penicillin/streptomycin as described previously. ${ }^{34}$ These cells were maintained exclusively in the laboratory of Xu Feng, Ph.D., in accordance with a materials transfer agreement. A plasmid, pMX-puro-Luc, was prepared by the insertion of firefly luciferase cDNA into the BamHI and NotI restriction sites of the pMX-puro retroviral vector. Then, 293GPG cells were transiently transfected with this vector using Lipofectamine Plus reagent (Invitrogen). Virus supernatants were collected at 48,72 and $96 \mathrm{~h}$ after transfection and then pooled. $\mathrm{C} 4-2 \mathrm{~B}$ cells were then infected with the viral supernatant for $24 \mathrm{~h}$ in the presence of $8 \mu \mathrm{g} / \mathrm{ml}$ Polybrene (hexadimethrine bromide). The infection medium was then replaced with cell culture medium and cells were allowed to recover for $24 \mathrm{~h}$ before selection with $2 \mu \mathrm{g} / \mathrm{ml}$ puromycin. 


\section{Viruses}

The wild-type human adenovirus serotype 5, Adwt300, was purchased from ATCC. The tropism-modified control virus Ad5-RGD has wild-type E1 and E3 regions as well as an RGD peptide in the HI loop of the fiber knob and was previously generated in our laboratory. ${ }^{22}$ The two unarmed control CRAds used in this study, Ad5- $\Delta 24$ and Ad5- $\Delta 24$ RGD, each have a 24-base pair deletion in the CR2 region of E1A and have been described previously. ${ }^{35}$ The tropism-modified control CRAd, Ad5- $\Delta 24 \mathrm{RGD}$, also has an RGD peptide in the HI loop of the fiber knob. The two armed CRAds used in this study, Ad5- $\Delta 24$-sOPG-Fc and Ad5- $\Delta$ 24-sOPG-Fc-RGD, each carry a transgene encoding the RANKL-binding domains of human OPG (amino acids 1-2015) fused to the Fc portion of human IgG1. ${ }^{28}$ The transgene is in place of the $\mathrm{E} 3 \mathrm{~B}$ region of the genome, under native expression control elements. The construction of these CRAds, as well as that of the E1-deleted replication-deficient control vectors Ad-CMV-sOPG-Fc-RGD and Ad-CMV-OPG-Fc-RGD, which expresses full-length OPG, has been detailed previously. ${ }^{22}$

\section{Expression of sOPG and Viral Genes}

Monolayers of $\mathrm{C} 4-2 \mathrm{~B}$ cells in 24-well plates were infected with Ad5- $\Delta 24-\mathrm{sOPG}-\mathrm{Fc}$, Ad5- $\Delta 24-\mathrm{sOPG}-\mathrm{Fc}-\mathrm{RGD}$ or Adwt300 at a multiplicity of infection (MOI) of 0.1 infectious units (IU) per cell in RPMI 1640 with 2\% (v/v) FBS. Cells were incubated for $1 \mathrm{~h}$ at $37^{\circ} \mathrm{C}$ before the infection mixtures were removed and replaced with serum-free growth medium with supplements. At various intervals post infection $(4,8$, 12,24 and $36 \mathrm{~h}$ ), medium was collected, and cell lysates were harvested by the addition of buffer RLT (RNeasy Mini Kit; Qiagen, Valencia, CA, USA) to the wells. Samples were stored at $-80{ }^{\circ} \mathrm{C}$ until they could be further processed.

Total cellular RNA was isolated from lysate samples using an RNeasy Mini Kit (Qiagen), according to the manufacturer's instructions. Purified RNA samples were then subjected to real-time quantitative reverse transcriptase PCR analysis using a LightCycler 480 system (Roche Diagnostics, Indianapolis, IN, USA). Samples from cells infected with the armed CRAds were assayed for the expression of sOPG-Fc, whereas samples from cells infected with Adwt300 were assayed for the expression of the $\mathrm{E} 3 \mathrm{~B}$ genes $14.7 \mathrm{k}$ and $\operatorname{RID} \beta$ (primer sequences previously published). ${ }^{22}$ All samples were analyzed for the expression of E3 gp19k, adenovirus death protein (ADP) and fiber. The expression of human glyceraldehyde-3-phosphate dehydrogenase was used as a control. Results are expressed as copy number/ng of total RNA.

\section{Secretion of sOPG-Fc}

Monolayers of LNCaP and C4-2B cells in 24-well plates were infected with Ad5- $\Delta 24-\mathrm{sOPG}-\mathrm{Fc}$ or Ad5- $\Delta 24-\mathrm{sOPG}-\mathrm{Fc}-\mathrm{RGD}$ as above, before the infection mixtures were removed and replaced with serum-free growth medium with supplements. Medium samples were collected at various intervals post infection $(24,36,48$ and $60 \mathrm{~h})$ and stored at $-80^{\circ} \mathrm{C}$. After the final time point, samples were thawed and concentrated to $1 / 10$ of the original volume using a Microcon centrifugal filter device (Millipore, Bedford, MA, USA) and centrifuged at $14000 \times g$. The presence of sOPG-Fc was determined by SDS-PAGE followed by immunoblotting using a goat antihuman OPG primary antibody (Sigma-Aldrich, St Louis, MO, USA) diluted 1:1000 and a rabbit anti-goat alkaline phosphatase-conjugated secondary antibody (Jackson ImmunoResearch, West Grove, PA, USA), diluted 1:4000. Blots were developed with 5-bromo-4-chloro-3-indolyl phosphate/nitro blue tetrazolium (Sigma-Aldrich).

\section{Viral DNA Replication in Cell Lines}

Monolayers of $\mathrm{C} 4-2 \mathrm{~B}$ cells in 24-well plates were infected with Adwt300, Ad5- $\Delta 24$, Ad5- $\Delta 24 R G D$, Ad5- $\Delta 24$-sOPG-Fc or Ad5- $\Delta 24-\mathrm{sOPG}-\mathrm{Fc}-\mathrm{RGD}$ at an MOI of $0.1 \mathrm{IU}$ per cell. At 2, 4 and 6 days post infection, $200 \mu \mathrm{l}$ samples of medium were collected and stored at $-20^{\circ} \mathrm{C}$ until further processing. DNA was then purified from the medium samples using a QIAamp DNA Blood Mini Kit (Qiagen). Samples were analyzed by quantitative real-time PCR on a LightCycler 480 system (Roche Diagnostics) for the presence of the Ad5 E4 gene (primer sequences previously published ${ }^{22}$ ), as an indicator of viral replication. ${ }^{36}$ Results are expressed as copy number/ng of total DNA.

\section{Cytopathic Effect}

To assay oncolytic potency qualitatively, monolayers of C42B, LNCaP and PC3 cells in 24-well plates were infected with Ad5- $\Delta 24-$ sOPG-Fc-RGD and each of the replicating control viruses at MOIs of 1, 0.1 and $0.01 \mathrm{IU}$ per cell. After 8 days, the viability of the cells was determined by staining the monolayers with $1 \%(\mathrm{w} / \mathrm{v})$ crystal violet (Fisher Scientific) in 70\% $(\mathrm{v} / \mathrm{v})$ ethanol for $1 \mathrm{~h}$. Plates were washed in tap water to remove excess dye.

\section{Osteoclast Formation}

The ability of the armed CRAds to inhibit osteoclast formation was assayed in both murine and human cells, using in vitro osteoclastogenesis assays that are detailed elsewhere and summarized here. ${ }^{22,33}$ In the murine system, bone marrow macrophages were isolated from 4- to 8-week-old female athymic nude Foxn $1^{\text {nu }}$ mice (Harlan, Indianapolis, IN, USA) and cocultured in a 10:1 ratio with ST2 murine bone marrow stromal cells in $\alpha$-MEM containing $10 \%(\mathrm{v} / \mathrm{v})$ FBS, $\quad 1 \times 10^{-8} \mathrm{M}$ 1,25-dihydroxyvitamin D3 (Biomol Research Laboratories, Plymouth Meeting, PA, USA) and $1 \times 10^{-6} \mathrm{M}$ dexamethasone (Sigma-Aldrich). After a $24 \mathrm{~h}$ recovery phase, porous $(0.4 \mu \mathrm{m}$ pore size) Transwell inserts $12 \mathrm{~mm}$ in diameter (Corning; Corning, NY, USA) containing monolayers of $\mathrm{C} 4-2 \mathrm{~B}$ cells that had been infected immediately before transfer at an MOI of $0.1 \mathrm{IU}$ per cell with each of the CRAds or Ad-CMV-OPG-Fc-RGD, diluted in RPMI 1640 with 2\% (v/v) FBS for $1 \mathrm{~h}$, were added to these 
cocultures. Cultures were maintained in $\alpha$-MEM supplemented with $10 \%(\mathrm{v} / \mathrm{v})$ FBS, $1 \times 10^{-8} \mathrm{M} 1,25$ dihydroxyvitamin D3 and $1 \times 10^{-6} \mathrm{M}$ dexamethasone.

In the human system, bone marrow macrophages were isolated from fresh human bone marrow purchased from Lonza (Lonza Walkersville, Walkersville, MD, USA) and prepared as previously described. ${ }^{37}$ These cells were plated in 24 -well plates and cultured in $\alpha$-MEM containing $10 \%$ FBS $(\mathrm{v} / \mathrm{v})$ supplemented with $10 \mathrm{ng} / \mathrm{ml}$ macrophage colonystimulating factor and $25 \mathrm{ng} / \mathrm{ml}$ recombinant human RANKL (R\&D Systems) for $48 \mathrm{~h}$ to allow attachment. Then, monolayers of $\mathrm{C} 4-2 \mathrm{~B}$ cells cultured on porous $(0.02 \mu \mathrm{m}$ pore size $) 10 \mathrm{~mm}$ diameter Anopore inserts (Nalge Nunc International; Rochester, NY, USA), which had been infected immediately before transfer with each of the CRAds or Ad-CMV-sOPG-Fc-RGD, as above, were transferred to the 24-well plates. The cultures were maintained in $\alpha$-MEM containing $10 \%$ FBS (v/v) supplemented with macrophage colony-stimulating factor and RANKL.

The cocultures were maintained in their respective osteoclastogenic media, with conditioned medium being collected from each well and replaced with $1 \mathrm{ml}$ fresh medium every 3 days. At the completion of each experiment, the inserts carrying prostate cancer cells were stained with crystal violet. Samples of conditioned medium from day 9 were assayed for the presence of the osteoclast-specific protein tartrate-resistant acid phosphatase $5 b^{38}$ as an indicator of osteoclast formation, using a MouseTRAP or BoneTRAP ELISA kit (Immuno-diagnostic Systems, Fountain Hills, AZ, USA) for murine and human osteoclasts, respectively.

\section{Murine Model of Prostate Cancer Bone Metastasis}

Animal experiments were conducted in accordance with federal and institutional guidelines for animal care. Osteoblastic lesions were established by the injection of $5 \times 10^{5} \mathrm{C} 4$ 2B-LUC cells into the left tibiae of 4- to 5-week-old male Fox Chase SCID beige mice (Harlan). ${ }^{39}$ Cells were prepared for injection by detachment with Versene followed by two washes in phosphate buffered saline (PBS) and a final resuspension in PBS at $2.5 \times 10^{7}$ cells $/ \mathrm{ml}$. Aliquots of $20 \mu \mathrm{l}\left(5 \times 10^{5}\right.$ cells $)$ of single cell suspension were loaded into BD Micro-Fine IV needle $(28 \mathrm{G})$ insulin syringes $(3 / 10 \mathrm{cc}$; BD Consumer Healthcare, Franklin Lakes, NJ, USA), which were kept on ice until the animals were ready for injection. Forty-five mice were anesthetized with $2 \%(\mathrm{v} / \mathrm{v})$ isoflurane (MWI, Meridian, ID, USA) gas at a flow rate of $0.5-11 / \mathrm{min}$ per mouse and were injected with cells in the proximal end of the left tibia. After 33 weeks, the mice were randomly divided into three treatment groups. Mice from two treatment groups were given intratibial injections of $2 \times 10^{6} \mathrm{IU}$ of either Ad5- $\Delta 24$ sOPG-Fc-RGD $(n=7)$ or Ad5- $\Delta 24 \mathrm{RGD}(n=7)$ in a total volume (TV) of $20 \mu \mathrm{l}$ of PBS. The third group of animals was injected with PBS only $(n=6)$. Three weeks after treatment, the mice were killed, as were three additional age-matched control naïve mice. The left tibia of each was dissected and preserved in $10 \%(\mathrm{w} / \mathrm{v})$ neutral buffered formalin (Fisher Scientific).

\section{Tomography}

For the determination of the $3 \mathrm{D}$ architecture of the trabecular bone, mouse tibiae were analyzed by micro computed tomography $(\mu \mathrm{CT})$, using a Scanco $\mu \mathrm{CT} 40$ desktop conebeam scanner (Scanco Medical AG, Brüttisellen, Switzerland). Tibiae were placed vertically in $12 \mathrm{~mm}$ diameter scanning holders. Scans were performed at the following settings: $6 \mu \mathrm{m}$ resolution, $70 \mathrm{kVp}, 114 \mu \mathrm{A}$ with an integration time of $200 \mathrm{~ms}$. Scans were automatically reconstructed into 2D slices, and the region of interest was outlined in each slice using the $\mu$ CT Evaluation Program (v5.0A, Scanco Medical).

The scan of the trabecular bone was performed below the growth plate, and each scan consisted of 209 slices, of which 100 were used for analysis. A region of interest was drawn on each of the 100 slices just inside the cortical bone, to include only the trabecular bone and marrow. Trabecular bone was thresholded at 247 , to distinguish it from the marrow. The $3 \mathrm{D}$ reconstruction was performed on the region of interest which only contained trabecular bone; no cortical bone was present in these regions of interest. Data were obtained on TV of the scanned area, the volume of trabecular bone (BV) within that area, BV/TV, trabecular bone density, trabecular number, separation and thickness.

\section{Statistical Analysis}

Student-Fisher $t$-tests were used to analyze data from in vitro osteoclast formation assays. For the tomography data, a Kruskal-Wallis test was used as a non-parametric alternative to ANOVA, to examine overall differences between the four groups. As all outcomes were marginally or highly significant overall, pairwise comparisons between groups were done via Wilcoxon two-sample tests to identify outcomes that were significantly different between treatments. There was no adjustment for multiple testing as these are hypothesis-generating experiments. In all analyses, differences were considered significant when $P \leq 0.05$.

\section{RESULTS \\ Characterization of a Tropism-Modified, sOPG-Fc-armed CRAd in Prostate Cancer Cells}

The genomes of the viruses used in this study are depicted in Figure 1. Ad5- $\Delta 24-\mathrm{sOPG}-\mathrm{Fc}-\mathrm{RGD}$ is a tropism-modified CRAd that expresses an sOPG-Fc fusion gene from the E3B region of the adenovirus genome. The sOPG-Fc transgene replaces the native $E 3 B$ genes $(\operatorname{RID} \alpha, \operatorname{RID} \beta$ and $14.7 \mathrm{k})$ and was placed under native gene expression control elements. This CRAd retains expression of the E3-11.6k ADP for efficient lysis of infected cells and includes an RGD-modified fiber knob for enhanced transduction of tumor cells, as well as a $\triangle 24$-modified $E 1 A$ gene for cancer-selective replication. An sOPG-Fc-armed CRAd with native tropism, 


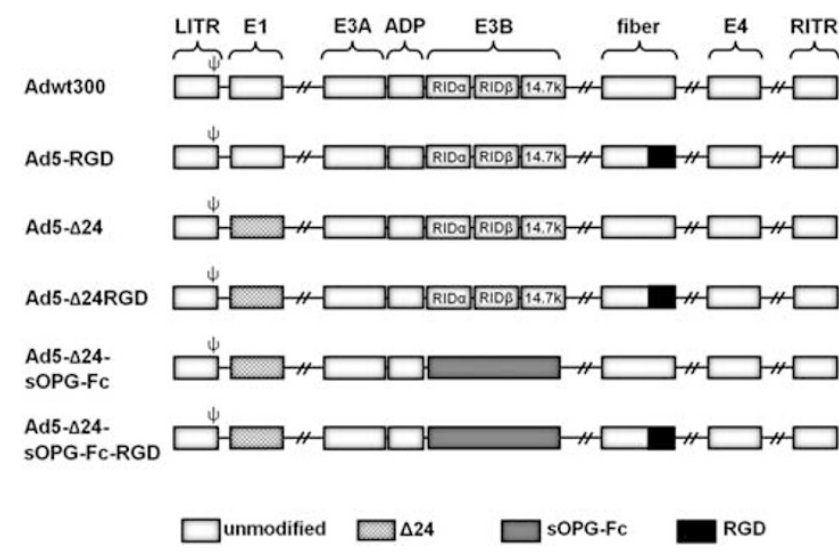

Figure 1 The genomes of the viruses used in this study are represented schematically. By convention, the adenovirus genome is depicted as having (from left to right): a left inverted terminal repeat (LITR) containing the packaging signal $(\psi)$, the early 1 (E1) gene, E3A region, $A D P, E 3 B$ region (containing the receptor internalization and degradation alpha (RID $\alpha), \operatorname{RID} \beta$ and 14.7k genes), fiber gene, E4 gene, and right inverted terminal repeat (RITR). For clarity, additional adenoviral genes are not shown. Modifications made to specific viruses, as indicated, include a 24-base pair deletion within E1 $(\Delta 24)$, replacement of E3B with an SOPG-Fc fusion gene and the inclusion of a RGD peptide within the knob domain of the fiber.

Ad5- $\Delta 24-$ sOPG-Fc, was included as a control for infectivity. Other control viruses included Ad5- $\Delta 24$ and Ad5- $\Delta 24$-RGD, unarmed CRAds with native and modified tropism, respectively. Ad5-RGD is a tropism-modified control virus that is otherwise syngeneic with the wild-type adenovirus Adwt300.

In a previous study, we demonstrated in breast cancer cells that the expression of sOPG-Fc from a CRAd mimicked that of the replaced native E3B genes, in both timing and amount. ${ }^{22}$ Hence, we first wished to confirm these findings in prostate cancer cells. We selected for analysis the C4-2B cell line, which is a subline of $\mathrm{LNCaP}$ with an enhanced propensity for bone metastasis in vivo. $\mathrm{C} 4-2 \mathrm{~B}$ cells were infected with Ad5- $\Delta 24-\mathrm{sOPG}-\mathrm{Fc}$, Ad5- $\Delta 24-\mathrm{sOPG}-\mathrm{Fc}-\mathrm{RGD}$ or Adwt300, and cell lysates were analyzed by quantitative reverse transcriptase PCR at multiple time points post infection. The sOPG-Fc transgene was expressed late in the infection cycle, at levels similar to that of the $14.7 \mathrm{k}$ gene from Ad300wt (Figure 2a). Also, the expression of ADP from Ad5$\Delta 24$-sOPG-Fc and Ad5- $\Delta 24$-sOPG-Fc-RGD is similar to that from Adwt300 in both timing and amount (Figure 2b). Together, these data indicate that the sOPG-Fc transgene is efficiently expressed in prostate cancer cells in a manner consistent with its placement in the adenoviral genome, and that the expression of surrounding viral genes is not altered.

To confirm that prostate cancer cells infected with the armed CRAds secrete sOPG-Fc into the medium, monolayers of both LNCaP and C4-2B cells were infected with Ad5- $\Delta 24$ sOPG-Fc-RGD or with Ad5- $\Delta 24-\mathrm{sOPG}-\mathrm{Fc}$. At multiple time points post infection, samples of conditioned medium were subjected to immunoblotting with an OPG-specific primary antibody. In samples from infected C4-2B cells, sOPG-Fc was detected in the medium beginning at $36 \mathrm{~h}$ post infection (Figure 2c). LNCaP cells released sOPG-Fc into the medium at $24 \mathrm{~h}$ and $36 \mathrm{~h}$ when infected with Ad5- $\Delta 24-\mathrm{sOPG}-\mathrm{Fc}-\mathrm{RGD}$ or with Ad5- $\Delta 24-\mathrm{sOPG}-\mathrm{Fc}$, respectively (Figure $2 \mathrm{~d}$ ). In both cell lines, sOPG-Fc protein increased in amount until $60 \mathrm{~h}$ post infection. These results confirm that prostate cancer cells infected with the armed CRAds efficiently secrete sOPG-Fc.

\section{Expression of sOPG-Fc does not Enhance the Oncolytic Potency of a CRAd in Prostate Cancer Cells}

We next sought to confirm that prostate cancer cells would support the replication of a CRAd armed with sOPG-Fc. Monolayers of $\mathrm{C} 4-2 \mathrm{~B}$ cells were infected with both the tropism-modified and -unmodified armed CRAds, their respective unarmed control CRAds, or with wild-type adenovirus. Conditioned medium was collected 2, 4 and 6 days post infection, and DNA isolated from the samples was analyzed by quantitative real-time PCR for the adenovirus E4 gene, as an indicator of viral replication. Both of the sOPG-Fc-armed CRAds replicated efficiently in the C4-2B cells, at levels similar to the unarmed CRAds and Adwt300 (Figure 3a). Thus, it is evident that the expression of sOPGFc from a CRAd does not enhance adenoviral replication in prostate cancer cells. To determine whether the expression of sOPG-Fc inhibits the ability of a CRAd to efficiently lyse infected prostate cancer cells, a panel of prostate cancer cells was infected with Ad5- $\Delta 24-\mathrm{sOPG}-\mathrm{Fc}-\mathrm{RGD}$ or control viruses. This panel included lines with low levels of CAR expression $\left(\mathrm{C} 4-2 \mathrm{~B}^{40}\right.$ and $\left.\mathrm{PC} 3^{41}\right)$ as well as a line expressing high levels of $\mathrm{CAR}\left(\mathrm{LNCaP}^{42}\right)$. After 8 days the monolayers were stained with crystal violet, in a qualitative assay for oncolytic potency. As indicated by the cleared wells resulting from viral oncolysis, all viruses were sufficiently potent to completely destroy the monolayers of each cell line at an MOI of 0.1 (Figure $3 \mathrm{~b}$ ). Although most viruses completely destroyed the monolayers at the lower MOI of 0.01 , the tropism-modified armed CRAd exhibited reduced oncolytic potency in comparison with its unarmed control. This result indicates that the expression of OPG does not enhance the potency of an armed CRAd in prostate cancer cells in vitro. This is not unexpected, as our hypothesis predicts that the additional antitumor effect of OPG expression would be manifested only in the bone microenvironment. Considered together with the viral replication data, these experiments demonstrate that the expression of sOPG-Fc from a CRAd does not enhance viral replication or oncolytic potency in prostate cancer cells.

\section{CRAds Armed with sOPG-Fc Inhibit Osteoclast Formation in vitro}

Both murine and human cell culture systems were used to determine whether prostate cancer cells infected with the armed CRAds would inhibit osteoclast formation while simultaneously being lysed by viral replication. Monolayers 

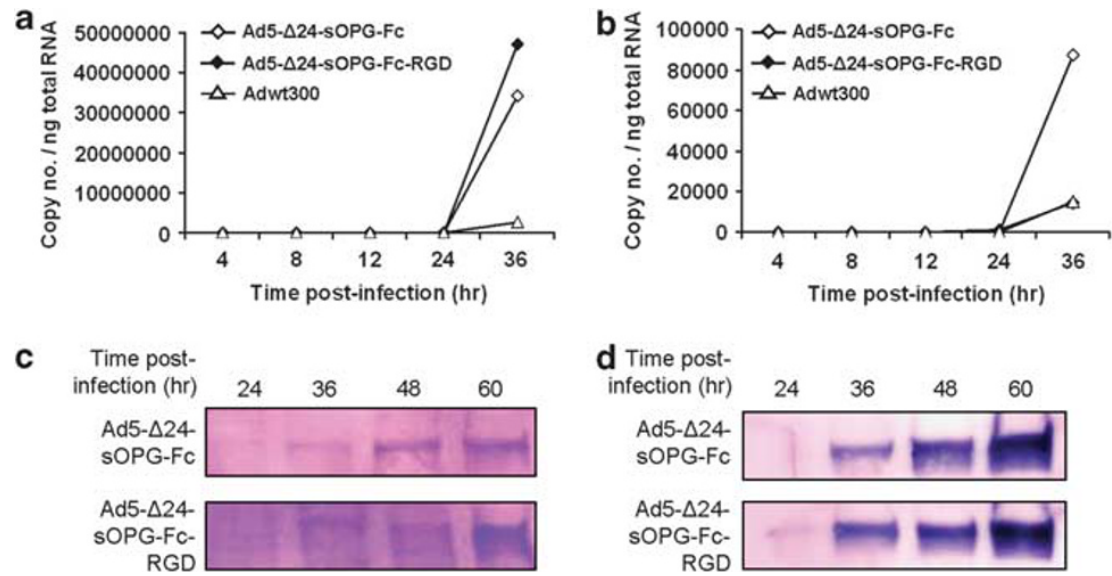

Figure 2 Characterization of armed CRAds. (a, b) C4-2B prostate cancer cells were infected with Ad5- $\Delta 24-\mathrm{sOPG}-\mathrm{Fc}$, Ad5- $\Delta 24-\mathrm{sOPG}-\mathrm{Fc}-\mathrm{RGD}$ or Adwt300. At the indicated times post infection, total cellular RNA was extracted and subjected to quantitative reverse transcriptase PCR to detect expression of: (a) the sOPG-Fc gene (for cells infected with Ad5- $\Delta 24-\mathrm{sOPG}-\mathrm{Fc}$ or Ad5- $\Delta 24-\mathrm{sOPG}-\mathrm{Fc}-\mathrm{RGD}$ ) or the $14.7 \mathrm{k}$ gene (for cells infected with Adwt300); and (b) the ADP gene. (c, d) Secretion of sOPG-Fc by infected C4-2B (c) and LNCaP (d) cells. At the indicated times post infection, conditioned medium was collected and subjected to immunoblot analysis using an anti-OPG primary antibody.

a

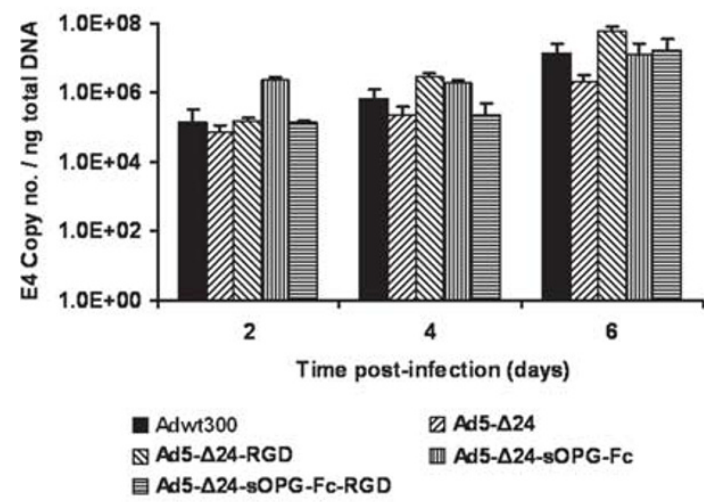

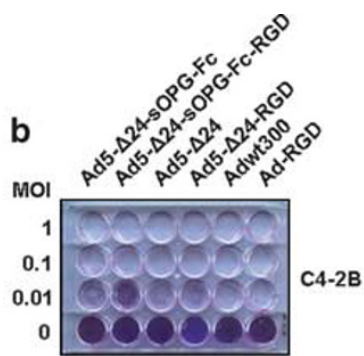
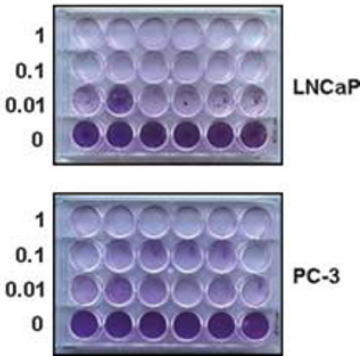

Figure 3 Oncolytic potency of the armed CRAds. (a) C4-2B prostate cancer cells were infected with Adwt300, Ad5- $\Delta 24$, Ad5- $\Delta 24 R G D$, Ad5- $\Delta 24-s O P G-F c$ or Ad5- $\Delta 24-$-sOPG-Fc-RGD. The conditioned culture medium was collected at 2, 4 and 6 days post infection. DNA was extracted and subjected to Q-PCR to detect the E4 gene as a measure of viral DNA replication. Results are the means \pm s.d. of duplicate determinations. Representative results of three separate experiments are shown. (b) a panel of prostate cancer cell lines was infected at the indicated MOls. Eight days post infection, viable cells were fixed and stained with crystal violet. Representative results of three separate experiments are shown.

of $\mathrm{C} 4-2 \mathrm{~B}$ cells were established on permeable cell culture inserts and then infected with the unarmed control CRAds, Ad5- $\Delta 24$ and Ad5- $\Delta 24 \mathrm{RGD}$, and both of the armed CRAds, Ad5- $\Delta 24$-sOPG-Fc or Ad5- $\Delta 24-$-sOPG-Fc-RGD. Additional wells infected with tropism-modified, E1-deleted replicationdeficient control vectors expressing OPG-Fc (murine experiment) or sOPG-Fc (human experiment) from the CMV promoter were included as controls for viral replication. The cell culture inserts containing the infected cells were then added either to cocultures of murine bone marrow macrophages and ST2 bone marrow stromal cells, or to cultures of human bone marrow macrophages in recombinant soluble RANKL-containing medium. In both experiments, cultures were maintained in osteoclastogenic medium and thus were expected to form osteoclasts within 7-10 days. On day 9, conditioned medium samples were analyzed by an ELISA for the osteoclast-specific protein TRAP5b as an indicator of osteoclast formation. In both the murine (Figure 4a) and the human (Figure 4b) experiments, wells containing armed CRAds with either tropism-modified or wild-type fibers inhibited the formation of osteoclasts relative to their respective unarmed control CRAd platforms $(P<0.05$ for all 

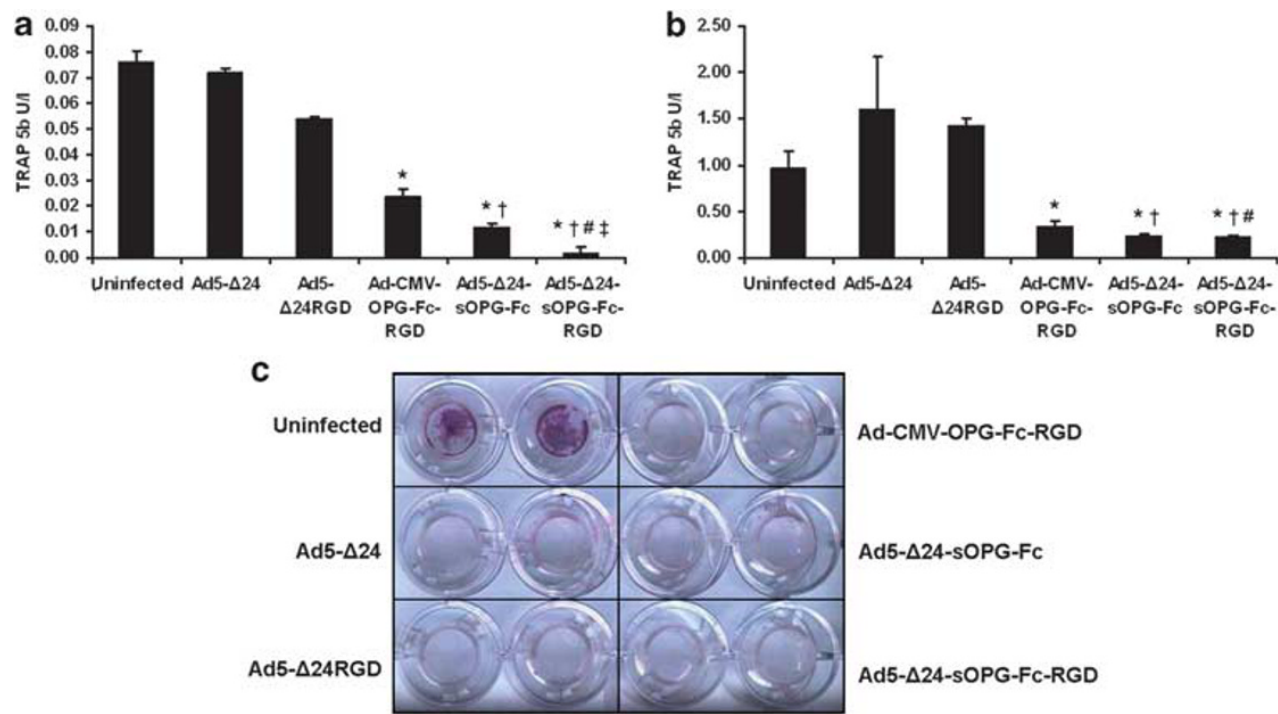

Figure 4 CRAds armed with sOPG-Fc simultaneously lyse prostate cancer cells and inhibit osteoclast formation in vitro. C4-2B cells were infected with the indicated adenoviruses and grown on inserts overlaying cocultures of murine osteoclast precursors and ST2 bone marrow stromal cells (a) or human osteoclast precursors and RANKL (b). At day 9, an ELISA was performed to detect TRAP5b, an osteoclast marker protein. Results are means \pm s.d. of duplicate determinations. Significant differences $(P<0.05)$ versus uninfected $(*)$, unarmed CRAd $(\dagger)$, non-replicative vector (\#), and armed CRAd with native tropism ( $\ddagger)$ are indicated. Representative results of two separate experiments are shown. (c) Viable prostate cancer cells on the inserts were fixed and stained with crystal violet.

pairwise comparisons) or to the replication-defective vectors. In addition, Ad5- $\Delta 24-$ sOPG-Fc-RGD inhibited the formation of osteoclasts to a greater extent than did Ad5- $\Delta 24$ sOPG-Fc in the murine cell coculture $(P<0.05)$. The monolayers containing the $\mathrm{C} 4-2 \mathrm{~B}$ cells were stained with crystal violet to assay cell viability. As shown for the murine experiment in Figure 4c, the cells were destroyed by the CRAds, indicating that tumor cell lysis occurs simultaneously with suppression of osteoclast formation. Overall, these findings support our hypothesis that an sOPG-Fc-armed CRAd can inhibit the growth of prostate cancer bone metastases by directly lysing tumor cells and by blocking the formation of osteoclasts.

\section{A Tropism-Modified CRAd Armed with sOPG-Fc Inhibits Prostate Cancer Bone Metastasis in vivo}

We next wished to demonstrate that the tropism-modified, armed CRAd, Ad5- $\Delta 24-$ sOPG-Fc-RGD, could inhibit the growth of prostate cancer bone metastases in vivo more effectively than its unarmed control CRAd, Ad5- 2 24RGD. Osteoblastic bone metastases were established in male SCID beige mice by the injection of C4-2B-LUC cells into the left tibiae. Subsequently, a subset of mice developed rapidly growing tumors that impaired locomotion and were removed from the study in accordance with institutional regulations. The remaining mice developed slow-growing tumors, which became palpable at $\sim 9$ weeks and continued to increase in size over the duration of the study. In this experiment, tumor size did not correlate with bioluminescence quantification, and thus the imaging was not continued. After 33 weeks, the mice were randomly divided into three cohorts and treated by the intratibial delivery of Ad5- $\Delta 24 \mathrm{RGD}$ or Ad5- $\Delta 24$ sOPG-Fc-RGD, or given PBS only as a control. Three weeks following treatment, the mice were killed and the tibiae were collected and analyzed by $\mu \mathrm{CT}$. The tibiae of three additional age-matched naïve mice were also collected and examined as examples of normal bone. The average ratio of trabecular bone volume to the total analyzed volume (BV/TV), surface area of trabecular bone and the density of the trabecular bone for each treatment group were then determined from the $\mu \mathrm{CT}$ data.

Mice in the PBS and Ad5- $\Delta 24 \mathrm{RGD}$ treatment groups exhibited a loss of trabecular bone, whereas mice treated with Ad5- $\Delta 24-s O P G-F c-R G D$ displayed a trabecular structure which more closely resembled that of the naïve control mice. Images of representative tibiae from each treatment group are shown in Figure 5. Comparison of group averages revealed a number of trends. Whereas naïve mice had a BV/TV ratio of 0.0644 , mice treated with PBS had ratio of 0.0223 and those treated with Ad5- $\Delta 24 \mathrm{RGD}$ had a ratio of 0.0246 , representing decreases of 65 and $62 \%$, respectively (Figure 6a). In contrast, the BV/TV ratio of Ad5- $\Delta 24-\mathrm{sOPG}$ Fc-RGD treated mice $(0.0496)$ was double that of the PBS and Ad5- $\triangle 24$ RGD groups, and had decreased only $23 \%$ vs the naive group. Similarly, as shown in Figure $6 \mathrm{~b}$, mice treated with the armed CRAd displayed a trabecular bone surface area, which more closely resembled the naïve mice $\left(2.6982\right.$ vs $\left.3.1104 \mathrm{~mm}^{2}\right)$ than did that of the PBS$\left(1.5437 \mathrm{~mm}^{2}\right)$ or Ad5- $\Delta 24 \mathrm{RGD}$-treated mice $\left(1.37 \mathrm{~mm}^{2}\right)$. Although the observed trends did not reach statistical 

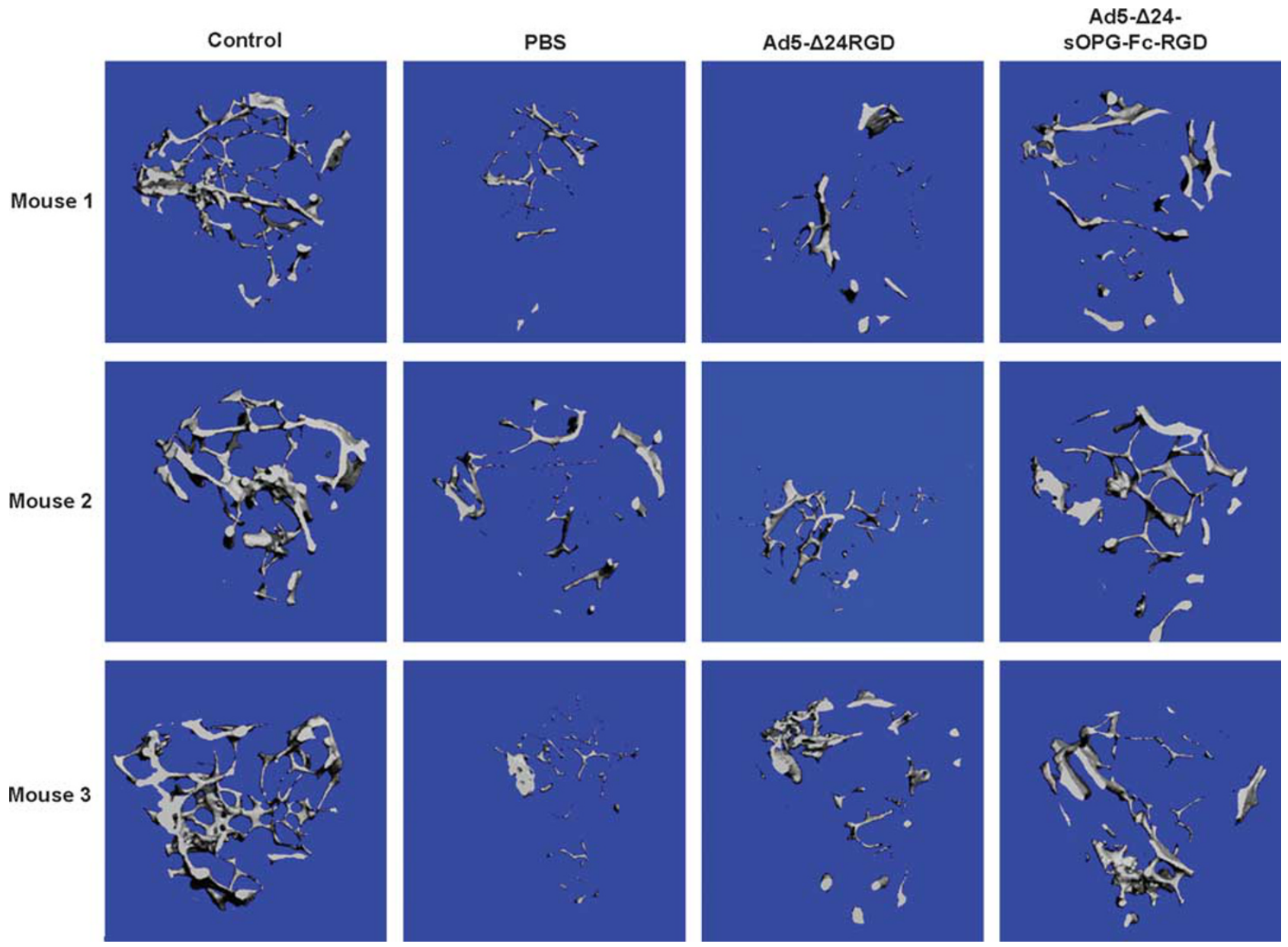

Figure 5 An sOPG-Fc-armed CRAd inhibits the progression of bone metastases of prostate cancer in vivo more effectively than does an unarmed CRAd. Intratibial tumors of C4-2B-LUC prostate cancer cells were established in SCID mice and treated with either Ad5- $\Delta 24-\mathrm{sOPG}-\mathrm{Fc}-\mathrm{RGD}$, Ad5- $\Delta 24 \mathrm{RGD}$ or PBS. Shown are $\mu \mathrm{CT}$ images of the proximal tibiae of three naïve control mice and three representative mice from each treatment group.

significance by Wilcoxon Two-Sample analysis, similar findings were also observed in the examination of trabecular bone density (not shown).

In aggregate, these data show that a CRAd armed with sOPG-Fc inhibits the progression of prostate cancer bone metastases and preserves normal bone architecture more effectively than does an unarmed control CRAd, but further titration of experimental conditions will need to be performed to maximize clinical efficacy.

\section{DISCUSSION}

We have designed an armed CRAd for bone metastases that targets both the metastatic tumor cell and the bone microenvironment. Prostate cancer commonly metastasizes to the skeleton ${ }^{1,2}$ where it relies on increased osteoclast activity. ${ }^{10,11}$ Thus, we hypothesized that an sOPG-Fc-armed CRAd would be effective against this disease. Here, we have confirmed that sOPG-Fc is expressed and secreted from prostate cancer cells infected with Ad5- $\Delta 24-\mathrm{sOPG}-\mathrm{Fc}-\mathrm{RGD}$, and that viral replication and tumor cell lysis are not enhanced by this expression. We showed that Ad5- $\Delta 24-\mathrm{sOPG}-\mathrm{Fc}-\mathrm{RGD}$ inhibits osteoclastogenesis while simultaneously lysing prostate cancer cells. Finally, we have shown that Ad5- $\Delta 24-\mathrm{sOPG}-$ Fc-RGD more effectively controls the growth of established bone lesions in vivo than does Ad5- $\Delta 24 \mathrm{RGD}$, by more effectively preserving the normal bone architecture. Altogether, these results supported our hypothesis that a CRAd armed with OPG can inhibit the growth of prostate cancer bone metastases by directly lysing tumor cells and by reducing osteoclast formation and activation.

We focused most of our studies on the $\mathrm{C} 4-2 \mathrm{~B}$ cell line, which is a bone metastatic derivative of the LNCaP line and establishes osteoblastic lesions in vivo. ${ }^{43,44}$ We also included for analysis LNCaP cells, which were derived from a lymph node metastasis, and PC3 cells, which were isolated from a bone metastasis. We observed similar levels of gene expression, viral replication and oncolytic potency between the tropism-modified and wild-type tropism CRAds in these cell lines. The fact that each of these lines expresses CAR, with C4- $2 \mathrm{~B}^{40}$ and PC3 cells ${ }^{41}$ expressing low but detectable levels 

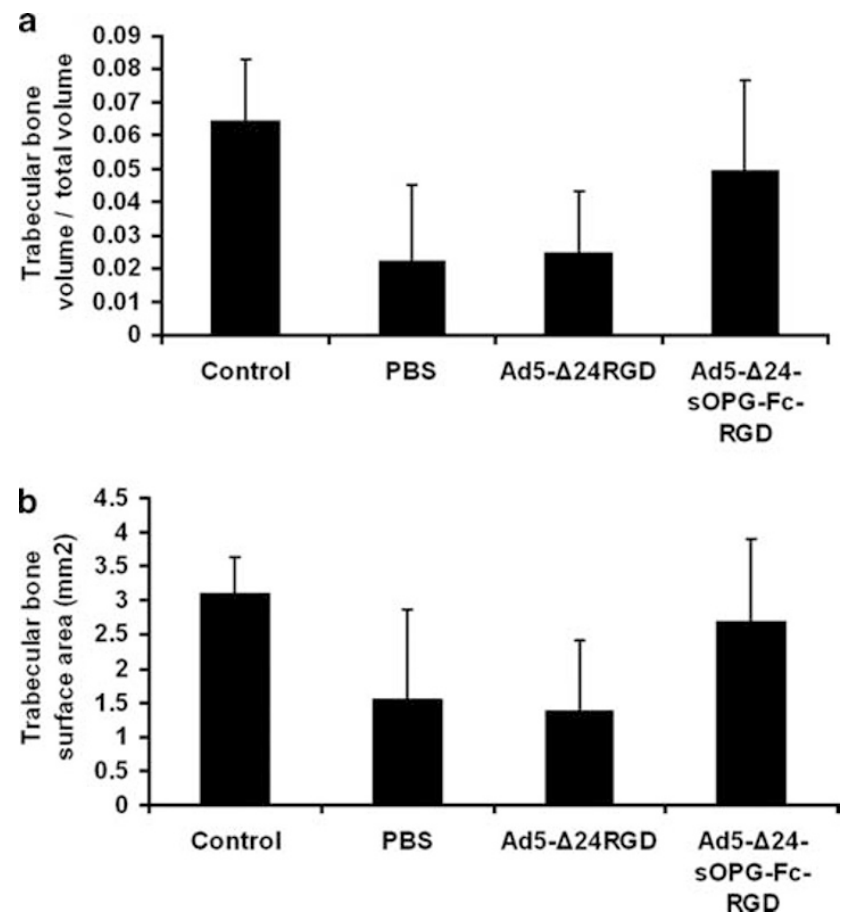

Figure 6 SCID mice bearing intratibial tumors of C4-2B-LUC were administered intratibial injections of Ad5- $\Delta 24-$ SOPG-Fc-RGD, Ad5- $\Delta 24 R G D$ or PBS and were compared to naïve controls. Tibiae were collected and subjected to $\mu \mathrm{CT}$. Analysis of $\mu \mathrm{CT}$ images was performed to determine the ratio of TV to trabecular bone volume (a) and trabecular bone surface area (b). Shown are the group means \pm s.d., $n=3$ (control), 6 (PBS), 7 (Ad- $\Delta 24 R G D)$ and 7 (Ad- $\Delta 24-s O P G-F c-R G D)$.

and LNCaP cells expressing high levels ${ }^{42}$ may explain these results. Although our in vitro experiments did not show a clear advantage in the use of the tropism-modified armed CRAd over its wild-type fiber control, a study by Rauen $e t$ al showed that the expression of CAR in prostate tumors is inversely correlated with tumor stage/aggressiveness. ${ }^{27}$ Interestingly, CAR was detected in the four bone metastasis specimens analyzed, but expression was not uniform. Regardless, the RGD tropism modification does not preclude binding of the virus to CAR. ${ }^{24}$ Therefore, the RGD-modified armed CRAd, with its expanded tropism, would likely be a more effective therapeutic than an isogenic CRAd with wild-type fibers. In our osteoclastogenesis experiments, cell death was observed in C4-2B cells infected with the non-replicative control vector. We speculate that this may have resulted from a toxic effect by some mechanism unrelated to replication; the reason for this cell death was not determined. Considered together with the oncolytic potency experiments, however, the data nonetheless show that an OPG-armed CRAd is capable of simultaneously destroying tumor cells by lysis while mediating an inhibition of osteoclastogenesis, consistent with our previous findings. ${ }^{22}$

For our in vivo experiment, we developed a $\mathrm{C} 4-2 \mathrm{~B}$ cell line which stably expresses luciferase. Our intention was to monitor tumor growth non-invasively by bioluminescence imaging. However, tumor luciferase expression did not correlate with tumor growth, and thus we were unable to rely upon imaging for tumor monitoring. This lack of correlation may have been due to the long time frame of the experiment, which may have allowed for a loss of luciferase expression. We observed the C4-2B tumors to be slow growing in vivo, which has been noted by others using this line. ${ }^{44-48}$ As in other intra-osseous murine models of prostate cancer bone metastases, progression is typically observed over periods of weeks to months, particularly in studies employing the LNCaP line and its derivatives. ${ }^{39,44,49-53}$ At the conclusion of our experiment, we observed extensive trabecular bone destruction rather than osteosclerosis, as indicated by an overall loss of trabecular bone in all treatment groups. This effect was also reported by Chanda et al, in a study demonstrating that intratibial tumors of $\mathrm{C} 4-2 \mathrm{~B}$ cells converted from an osteoblastic to an osteolytic phenotype after 6 months in vivo. ${ }^{48}$ In a rat model of prostate cancer bone metastasis, Lynch et al showed that osteoclast numbers increased in mixed lesions up to 4 weeks, when the experiment was concluded. ${ }^{54}$ This suggests that after an initial osteoblastic phase, the constant upregulation of osteoclast activity leads to an overall loss of bone in mature lesions. The biphasic growth of prostate cancer bone metastases may account for the contradictory roles of OPG in their development. Prostate cancer cell lines, including $\mathrm{PC} 3, \mathrm{LNCaP}^{29}$ and $\mathrm{C} 4-2 \mathrm{~B},{ }^{55}$ express OPG. This expression may promote tumor cell survival and bone formation initially, but is nonetheless insufficient to prevent the eventual loss of bone in mature lesions.

We intended to treat well-established tumors and therefore administered treatment at week 33. By treating established tumors, this was a more challenging model but one that more closely represented a clinical scenario. A variety of studies have examined the potential of OPG for the treatment of bone metastases of prostate cancer in murine models. These include studies in which OPG expression within a prostate cancer bone lesion inhibited both osteoblastic ${ }^{19}$ and osteolytic $^{48}$ tumor progression and others involving the use of recombinant $\mathrm{OPG}^{17,18,20,53,56}$ in intratibial models of prostate bone metastases. In these models, which include the osteolytic PC3 model ${ }^{14,20}$ and the osteoblastic $\mathrm{LNCaP}^{18}$ and C4-2B ${ }^{17}$ models, it has consistently been shown that the administration of recombinant OPG to tumor-bearing mice significantly reduces the growth of established intratibial lesions, but does not inhibit the growth of prostate cancer cells in vitro or the growth of subcutaneous xenografts. Two reports have implicated extracellular calcium in the growth of prostate cancer bone metastases, ${ }^{57,58}$ suggesting that the inhibition of bone resorption by factors such as OPG, by means of calcium depletion, may contribute to a reduction in prostate cancer bone metastasis. However, because OPG does not affect proliferation of prostate cancer cells, and is not directly cytotoxic, it is unlikely that OPG administration alone would be sufficient to eliminate established metastases. Regarding safety, it is possible that the expression of OPG 
from a CRAd could influence the surrounding normal bone, with the most likely effect being a transient increase in bone formation. However, OPG was safely given to multiple myeloma and breast cancer patients in a Phase I trial..$^{59}$

For both tumor cell implantation and delivery of the CRAds, we utilized intratibial injection. ${ }^{39}$ This method is frequently employed in models of prostate cancer bone metastasis, as systemic and orthotopic prostate cancer models do not efficiently establish bone metastases. ${ }^{4,60}$ This delivery method may have limited the antitumor effect that we were able to achieve, as the small size of the intratibial compartment limited the amount of virus that could be injected. Intratibial injection was a practical necessity, however, because the systemic delivery of adenovirus is unfeasible from a clinical standpoint, as intravenously delivered adenoviruses are largely sequestered by the liver. ${ }^{61}$ As work continues in the field of adenoviral targeting, this problem may be overcome in the future. In the $\mu \mathrm{CT}$ analysis of the tumor-bearing tibiae, we observed a wide variability within treatment groups that prevented the observed trends from reaching statistical significance. This limitation of the intratibial tumor model has been reported by others. ${ }^{62}$ Although the observed trends were consistent across all measurements, larger treatment groups would have made statistical significance easier to attain. However, it is also likely that, due to inherently variable growth rates of tumors in vivo, unacceptably large numbers of mice might have been necessary, suggesting that better animal models are an additional requirement to move the field forward. Finally, this study was designed to examine certain end points. An expanded study in which samples are collected at specific time points to examine dynamic changes in viral replication and tumor/bone interactions would reveal valuable information that would guide the clinical application of this CRAd.

We have demonstrated the potential utility of an sOPGFc-armed CRAd as a treatment for prostate cancer bone metastases. A number of studies have been published regarding the use of oncolytic viruses for prostate cancer and have been reviewed by Fukuhara et al. ${ }^{63}$ In particular, studies involving CRAds in models of prostate cancer bone metastasis, ${ }^{64,65}$ including one employing the $\mathrm{C} 4-2 \mathrm{~B}$ model $^{66}$ and one in which CRAds armed with a soluble transforming growth factor beta receptor II-Fc fusions are used in a PC3 model ${ }^{67}$ have underscored the potential for this treatment strategy. This report, however, is the first to evaluate an armed CRAd designed specifically for the bone microenvironment in a model of prostate cancer bone metastases. Furthermore, it is likely that this CRAd, Ad5- $\Delta 24-\mathrm{sOPG}$ Fc-RGD, will also be effective against other malignancies that metastasize to the skeleton such as lung, thyroid, or renal carcinomas.

\section{ACKNOWLEDGEMENTS}

We thank Xingsheng Li, and Maria S Johnson, at the Small Animal Bone Phenotyping Core facility in the UAB Center for Metabolic Bone Disease for technical assistance with the $\mu \mathrm{CT}$ analysis. This work was supported by National Institutes of Health Grants R01 CA108585 and T32 CA075930.

\section{DISCLOSURE/CONFLICT OF INTEREST}

The authors declare that equity in VectorLogics is held by JT Douglas.

1. Bubendorf L, Schopfer A, Wagner U, et al. Metastatic patterns of prostate cancer: an autopsy study of 1,589 patients. Hum Pathol 2000;31:578-583.

2. Coleman RE. Clinical features of metastatic bone disease and risk of skeletal morbidity. Clin Cancer Res 2006;12:6243s-6249ss.

3. Roudier MP, Vesselle H, True LD, et al. Bone histology at autopsy and matched bone scintigraphy findings in patients with hormone refractory prostate cancer: the effect of bisphosphonate therapy on bone scintigraphy results. Clin Exp Metastasis 2003;20:171-180.

4. Coleman RE. Metastatic bone disease: clinical features, pathophysiology and treatment strategies. Cancer Treat Rev 2001; 27:165-176.

5. James ND, Bloomfield D, Luscombe C. The changing pattern of management for hormone-refractory, metastatic prostate cancer. Prostate Cancer Prostatic Dis 2006;9:221-229.

6. Alemany R, Balague C, Curiel DT. Replicative adenoviruses for cancer therapy. Nat Biotechnol 2000;18:723-727.

7. Kirn D. Clinical research results with dl1520 (Onyx-015), a replicationselective adenovirus for the treatment of cancer: what have we learned? Gene Ther 2001;8:89-98.

8. Cody JJ, Douglas JT. Armed replicating adenoviruses for cancer virotherapy. Cancer Gene Ther 2009;16:473-488.

9. Mundy GR. Metastasis to bone: causes, consequences and therapeutic opportunities. Nat Rev Cancer 2002;2:584-593.

10. Roato I, D'Amelio P, Gorassini E, et al. Osteoclasts are active in bone forming metastases of prostate cancer patients. PLoS One 2008;3:e3627.

11. Inoue H, Nishimura K, Oka D, et al. Prostate cancer mediates osteoclastogenesis through two different pathways. Cancer Lett 2005;223:121-128.

12. Zhang J, Dai J, Yao Z, et al. Soluble receptor activator of nuclear factor kappaB Fc diminishes prostate cancer progression in bone. Cancer Res 2003;63:7883-7890.

13. Yonou H, Kanomata N, Goya M, et al. Osteoprotegerin/osteoclastogenesis inhibitory factor decreases human prostate cancer burden in human adult bone implanted into nonobese diabetic/severe combined immunodeficient mice. Cancer Res 2003;63:2096-2102.

14. Whang PG, Schwarz EM, Gamradt SC, et al. The effects of RANK blockade and osteoclast depletion in a model of pure osteoblastic prostate cancer metastasis in bone. J Orthop Res 2005;23:1475-1483.

15. Simonet WS, Lacey DL, Dunstan CR, et al. Osteoprotegerin: a novel secreted protein involved in the regulation of bone density. Cell 1997;89:309-319.

16. Udagawa N, Takahashi N, Yasuda H, et al. Osteoprotegerin produced by osteoblasts is an important regulator in osteoclast development and function. Endocrinology 2000;141:3478-3484.

17. Zhang J, Dai J, Qi Y, et al. Osteoprotegerin inhibits prostate cancerinduced osteoclastogenesis and prevents prostate tumor growth in the bone. J Clin Invest 2001;107:1235-1244.

18. Kiefer JA, Vessella RL, Quinn JE, et al. The effect of osteoprotegerin administration on the intra-tibial growth of the osteoblastic LuCaP 23.1 prostate cancer xenograft. Clin Exp Metastasis 2004;21:381-387.

19. Corey E, Brown LG, Kiefer JA, et al. Osteoprotegerin in prostate cancer bone metastasis. Cancer Res 2005;65:1710-1718.

20. Armstrong AP, Miller RE, Jones JC, et al. RANKL acts directly on RANKexpressing prostate tumor cells and mediates migration and expression of tumor metastasis genes. Prostate 2008;68:92-104.

21. Miller RE, Roudier M, Jones J, et al. RANK ligand inhibition plus docetaxel improves survival and reduces tumor burden in a murine model of prostate cancer bone metastasis. Mol Cancer Ther 2008;7:2160-2169.

22. Cody JJ, Rivera AA, Lyons GR, et al. Arming a replicating adenovirus with osteoprotegerin reduces the tumor burden in a murine model of osteolytic bone metastases of breast cancer. Cancer Gene Ther 2010;17:893-905. 
23. Fueyo J, Gomez-Manzano C, Alemany R, et al. A mutant oncolytic adenovirus targeting the $\mathrm{Rb}$ pathway produces anti-glioma effect in vivo. Oncogene 2000;19:2-12.

24. Dmitriev I, Krasnykh V, Miller CR, et al. An adenovirus vector with genetically modified fibers demonstrates expanded tropism via utilization of a coxsackievirus and adenovirus receptor-independent cell entry mechanism. J Virol 1998;72:9706-9713.

25. Cooper $\mathrm{CR}$, Chay $\mathrm{CH}$, Pienta KJ. The role of alpha(v)beta(3) in prostate cancer progression. Neoplasia 2002;4:191-194.

26. Bisanz K, Yu J, Edlund M, et al. Targeting ECM-integrin interaction with liposome-encapsulated small interfering RNAs inhibits the growth of human prostate cancer in a bone xenograft imaging model. Mol Ther 2005;12:634-643

27. Rauen KA, Sudilovsky D, Le $J$, et al. Expression of the coxsackie adenovirus receptor in normal prostate and in primary and metastatic prostate carcinoma: potential relevance to gene therapy. Cancer Res 2002;62:3812-3818.

28. Emery JG, McDonnell $P$, Burke $M B$, et al. Osteoprotegerin is a receptor for the cytotoxic ligand TRAIL. J Biol Chem 1998;273:14363-14367.

29. Holen I, Croucher PI, Hamdy FC, et al. Osteoprotegerin (OPG) is a survival factor for human prostate cancer cells. Cancer Res 2002;62:1619-1623.

30. Horoszewicz JS, Leong SS, Chu TM, et al. The LNCaP cell line-a new model for studies on human prostatic carcinoma. Prog Clin Biol Res 1980;37:115-132.

31. Horoszewicz JS, Leong SS, Kawinski E, et al. LNCaP model of human prostatic carcinoma. Cancer Res 1983:43:1809-1818.

32. Kaighn ME, Narayan KS, Ohnuki $\mathrm{Y}$, et al. Establishment and characterization of a human prostatic carcinoma cell line (PC-3) Invest Urol 1979;17:16-23.

33. Udagawa $\mathrm{N}$, Takahashi $\mathrm{N}$, Akatsu $\mathrm{T}$, et al. The bone marrow-derived stromal cell lines MC3T3-G2/PA6 and ST2 support osteoclast-like cell differentiation in cocultures with mouse spleen cells. Endocrinology 1989;125:1805-1813.

34. Ory DS, Neugeboren BA, Mulligan RC. A stable human-derived packaging cell line for production of high titer retrovirus/vesicular stomatitis virus G pseudotypes. Proc Natl Acad Sci USA 1996;93: $11400-11406$.

35. Suzuki K, Alemany $R$, Yamamoto $M$, et al. The presence of the adenovirus $\mathrm{E} 3$ region improves the oncolytic potency of conditionally replicative adenoviruses. Clin Cancer Res 2002;8:3348-3359.

36. Rivera AA, Wang M, Suzuki $K$, et al. Mode of transgene expression after fusion to early or late viral genes of a conditionally replicating adenovirus via an optimized internal ribosome entry site in vitro and in vivo. Virology 2004;320:121-134.

37. Cody JJ, Rivera AA, Liu J, et al. A simplified method for the generation of human osteoclasts in vitro. Int J Biochem Mol Biol 2011;2:183-189.

38. Halleen JM, Alatalo SL, Suominen $\mathrm{H}$, et al. Tartrate-resistant acid phosphatase 5b: a novel serum marker of bone resorption. J Bone Miner Res 2000;15:1337-1345.

39. Corey E, Quinn JE, Bladou F, et al. Establishment and characterization of osseous prostate cancer models: intra-tibial injection of human prostate cancer cells. Prostate 2002;52:20-33.

40. Kasman L, Onicescu G, Voelkel-Johnson C. Histone deacetylase inhibitors restore cell surface expression of the coxsackie adenovirus receptor and enhance CMV promoter activity in castration-resistant prostate cancer cells. Prostate Cancer 2012;2012:137163.

41. Pandha HS, Stockwin LH, Eaton J, et al. Coxsackie B and adenovirus receptor, integrin and major histocompatibility complex class I expression in human prostate cancer cell lines: implications for gene therapy strategies. Prostate Cancer Prostatic Dis 2003;6:6-11.

42. Seki T, Dmitriev I, Suzuki K, et al. Fiber shaft extension in combination with $\mathrm{HI}$ loop ligands augments infectivity for CAR-negative tumor targets but does not enhance hepatotropism in vivo. Gene Ther 2002;9:1101-1108.

43. Thalmann GN, Anezinis PE, Chang SM, et al. Androgen-independent cancer progression and bone metastasis in the LNCaP model of human prostate cancer. Cancer Res 1994;54:2577-2581.

44. Wu TT, Sikes RA, Cui Q, et al. Establishing human prostate cancer cell xenografts in bone: induction of osteoblastic reaction by prostatespecific antigen-producing tumors in athymic and SCID/bg mice using LNCaP and lineage-derived metastatic sublines. Int J Cancer 1998;77:887-894.
45. Hall CL, Bafico A, Dai J, et al. Prostate cancer cells promote osteoblastic bone metastases through Wnts. Cancer Res 2005;65:7554-7560.

46. Kitagawa Y, Dai J, Zhang J, et al. Vascular endothelial growth factor contributes to prostate cancer-mediated osteoblastic activity. Cancer Res 2005;65:10921-10929.

47. Rubin J, Fan X, Rahnert J, et al. IGF-I secretion by prostate carcinoma cells does not alter tumor-bone cell interactions in vitro or in vivo. Prostate 2006:66:789-800.

48. Chanda D, Isayeva T, Kumar S, et al. Therapeutic potential of adult bone marrow-derived mesenchymal stem cells in prostate cancer bone metastasis. Clin Cancer Res 2009;15:7175-7185.

49. Fizazi $\mathrm{K}$, Yang J, Peleg $\mathrm{S}$, et al. Prostate cancer cells-osteoblast interaction shifts expression of growth/survival-related genes in prostate cancer and reduces expression of osteoprotegerin in osteoblasts. Clin Cancer Res 2003;9:2587-2597.

50. Burton DW, Geller J, Yang $M$, et al. Monitoring of skeletal progression of prostate cancer by GFP imaging, X-ray, and serum OPG and PTHrP. Prostate 2005;62:275-281.

51. Yang M, Burton DW, Geller J, et al. The bisphosphonate olpadronate inhibits skeletal prostate cancer progression in a green fluorescent protein nude mouse model. Clin Cancer Res 2006;12:2602-2606.

52. Bonfil RD, Dong Z, Trindade Filho JC, et al. Prostate cancer-associated membrane type 1-matrix metalloproteinase: a pivotal role in bone response and intraosseous tumor growth. Am J Pathol 2007;170: 2100-2111.

53. Morrissey C, Kostenuik PL, Brown LG, et al. Host-derived RANKL is responsible for osteolysis in a C4-2 human prostate cancer xenograft model of experimental bone metastases. BMC Cancer 2007;7:148.

54. Lynch CC, Hikosaka A, Acuff HB, et al. MMP-7 promotes prostate cancer-induced osteolysis via the solubilization of RANKL. Cancer Cell 2005:7:485-496

55. Lin DL, Tarnowski CP, Zhang J, et al. Bone metastatic LNCaP-derivative C4-2B prostate cancer cell line mineralizes in vitro. Prostate 2001;47:212-221.

56. Quinn JE, Brown LG, Zhang J, et al. Comparison of Fc-osteoprotegerin and zoledronic acid activities suggests that zoledronic acid inhibits prostate cancer in bone by indirect mechanisms. Prostate Cancer Prostatic Dis 2005;8:253-259.

57. Li X, Liao J, Park SI, et al. Drugs which inhibit osteoclast function suppress tumor growth through calcium reduction in bone. Bone 2011;48:1354-1361.

58. Liao J, Schneider A, Datta NS, et al. Extracellular calcium as a candidate mediator of prostate cancer skeletal metastasis. Cancer Res 2006;66:9065-9073.

59. Body JJ, Greipp P, Coleman RE, et al. A phase I study of AMGN-0007, a recombinant osteoprotegerin construct, in patients with multiple myeloma or breast carcinoma related bone metastases. Cancer 2003;97:887-892.

60. Singh AS, Figg WD. In vivo models of prostate cancer metastasis to bone. J Urol 2005;174:820-826.

61. Glasgow JN, Everts M, Curiel DT. Transductional targeting of adenovirus vectors for gene therapy. Cancer Gene Ther 2006;13:830-844.

62. Canon JR, Roudier M, Bryant R, et al. Inhibition of RANKL blocks skeletal tumor progression and improves survival in a mouse model of breast cancer bone metastasis. Clin Exp Metastasis 2008;25:119-129.

63. Fukuhara $\mathrm{H}$, Homma $\mathrm{Y}$, Todo $\mathrm{T}$. Oncolytic virus therapy for prostate cancer. Int J Urol 2010;17:20-30.

64. Matsubara S, Wada Y, Gardner TA, et al. A conditional replicationcompetent adenoviral vector, Ad-OC-E1a, to cotarget prostate cancer and bone stroma in an experimental model of androgen-independent prostate cancer bone metastasis. Cancer Res 2001;61:6012-6019.

65. Sandberg L, Papareddy P, Silver J, et al. Replication-competent Ad11p vector (RCAd11p) efficiently transduces and replicates in hormonerefractory metastatic prostate cancer cells. Hum Gene Ther 2009;20:361-373.

66. Li Y, Kacka M, Thompson M, et al. Conditionally replicating adenovirus therapy utilizing bone sialoprotein promoter (Ad-BSP-E1a) in an in vivo study of treating androgen-independent intraosseous prostate cancer. Urol Oncol 2011;29:624-633.

67. Hu Z, Gupta J, Zhang Z, et al. Systemic delivery of oncolytic adenoviruses targeting transforming growth factor- $\beta$ inhibits established bone metastasis in a prostate cancer mouse model. Hum Gene Ther 2012;8:871-882. 